\title{
Increased Expression of Brain-Derived Neurotrophic Factor Preserves Retinal Function and Slows Cell Death from Rhodopsin Mutation or Oxidative Damage
}

\author{
Godwin Okoye, ${ }^{1}$ Joelle Zimmer, ${ }^{1}$ Jennifer Sung, ${ }^{1}$ Peter Gehlbach, ${ }^{1}$ Tye Deering, ${ }^{1}$ Hiroyuki Nambu, ${ }^{1}$ Sean Hackett, ${ }^{1}$ \\ Michele Melia, ${ }^{1}$ Noriko Esumi, ${ }^{1}$ Donald J. Zack, ${ }^{1,2}$ and Peter A. Campochiaro ${ }^{1}$ \\ Departments of ${ }^{1}$ Ophthalmology and Neuroscience and ${ }^{2}$ Molecular Biology and Genetics, and Institute of Genetic Medicine, The Johns Hopkins University \\ School of Medicine, Baltimore, Maryland 21287-9277
}

\begin{abstract}
There are no effective treatments for inherited retinal degenerations, which are prevalent causes of visual disability. Several proteins promote the survival of various types of neurons, and increasing expression of one or more of these survival factors is a promising strategy for a new treatment. Studies examining the effects of intravitreous injections of brain-derived neurotrophic factor (BDNF) in models of inherited retinal degenerations have suggested that BDNF has little survival-promoting activity for photoreceptors. In this study, we generated double transgenic mice with doxycycline-inducible expression of BDNF in the retina. In a model of primary rod photoreceptor degeneration, expression of BDNF resulted in significant delay in photoreceptor cell death and maintenance of retinal function assessed by electroretinogram recordings. Expression of BDNF also caused strong protection of photoreceptors from oxidative damage-induced cell death. These data suggest that continuous expression of BDNF, unlike intravitreous injections, results in morphologic and functional benefit in animal models of inherited retinal degeneration. Double transgenic mice with inducible expression of survival factors provide valuable tools for selection of survival factor candidates for gene therapy.
\end{abstract}

Key words: BDNF; gene transfer; inducible transgenics; neurotrophic factors; oxidative damage; retinal degenerations

\section{Introduction}

Inherited retinal dystrophies are a prevalent cause of severe loss of vision for which there are no effective treatments. Survival factors provide a promising approach, and several have been tested by different routes of delivery in different animal models of retinal degeneration. The first approach was to inject recombinant survival factor proteins into the vitreous of rats or mice with retinal degenerations (Faktorovich et al., 1990, 1992; LaVail et al., 1992). These studies demonstrated that FGF2 slows photoreceptor degeneration in Royal College of Surgeon rats (Faktorovich et al., 1990), and that multiple survival factors, including FGF2, FGF1, BDNF, and CNTF, decrease light-induced photoreceptor cell death (LaVail et al., 1992). Intravitreous injections of CNTF analogs in mouse models of inherited retinal degenerations resulted in evidence of benefit in some types of degenerations, but there was little effect from injection of BDNF or FGF2 (LaVail et al., 1998). It was concluded that analogs of CNTF have the best survival-promoting activity in inherited degenerations in mice and provide the best candidates for treatment in humans. This conclusion has been supported by studies in cats with an autosomal dominant rod-cone dystrophy, in which intravitreous injec-

Received Dec. 11, 2002; revised March 3, 2003; accepted March 4, 2003.

This work was supported by a grant from the Foundation Fighting Blindness; National Eye Institute Grants EY05951, K08EY13420, and P30EY1765; a Lew R. Wasserman Merit Award (P.A.C.); unrestricted funds from Research to Prevent Blindness; a grant from Dr. and Mrs. William Lake; and a grant from the Steinbach Foundation (P.A.C. and D.J.Z.).P.A.C. is the George S. and Dolores Dore Eccles Professor of Ophthalmology. D. J. Z. is the Guerrieri Professor of Ophthalmology. We thank Dr. Freda Miller for providing CDNA for BDNF.

Correspondence should be addressed to Dr. Peter A. Campochiaro, Maumenee 719, The Johns Hopkins University School of Medicine, 600 North Wolfe Street, Baltimore, MD 21287-9277. E-mail: pcampo@jhmi.edu.

Copyright $\odot 2003$ Society for Neuroscience $\quad$ 0270-6474/03/234164-09\$15.00/0 tions of CNTF, but not BDNF, provided benefit (Chong et al., 1999).

Another approach has been to use gene transfer to express survival factors in the retinas and/or retinal pigmented epithelial cells of mice or rats with retinal degenerations. In two mouse models of recessive primary rod cell degeneration, retinal degeneration (rd) mice, which are homozygous for a mutation in the $\beta$ subunit of phosphodiesterase, and $\operatorname{Prph} 2 \mathrm{Rd}^{2 / \mathrm{Rd} 2}$ mice, which have a recessive mutation in peripherin (Travis et al., 1989; Connell et al., 1991), subretinal injection of adenoviral (Ad) vectors encoding a secretable form of CNTF delayed photoreceptor cell death and loss of electroretinogram (ERG) a-wave and b-wave amplitudes (Cayouette and Gravel, 1997; Cayouette et al., 1998). However, another study found that intravitreous injection of adenoassociated virus (AAV) vectors encoding secretable CNTF in Prph2Rd $2 / R d 2$ mice or two transgenic (Tg) rat models with photoreceptor degeneration (caused by expression of two different rhodopsin mutants) resulted in delay of photoreceptor cell death and more rapid deterioration of retinal function based on ERG recordings (Liang et al., 2001). Because these two studies show that a survival factor is capable of slowing photoreceptor cell death in $\operatorname{Prph} 2 \mathrm{Rd}^{2 / R d} 2$ mice, despite failure to observe any slowing of cell death in $139 \mathrm{Prph}_{2} \mathrm{Rd}^{2 / \mathrm{Rd} 2}$ mice given intravitreous injections of single or combinations of survival factors (LaVail et al., 1998), it is clear that negative results obtained with the intravitreous injection strategy should be viewed with caution. The gene transfer strategy probably provides a better approach for selecting promising survival factor candidates, but still has problems, as shown by the discrepancy in Prph2Rd ${ }^{2 / R d} 2$ mice noted above; Ad-vectored CNTF resulted in preservation of ERG am- 
plitudes, whereas AAV-vectored CNTF resulted in accelerated deterioration of ERG amplitudes. There are several possible explanations for this discrepancy, such as vector-related effects, differences in duration of CNTF expression, and differences in cell types transduced, but regardless of the cause, the conflicting results indicate that alternative strategies for assessing potential beneficial or deleterious effects of survival factors would be useful.

Generation of transgenic mice with inducible expression of survival factors in the retina provides a strategy that avoids vector-related variables in studies aimed at assessing the effects of survival factors in the retina. In this study, we have used this strategy to assess the effects of BDNF in the retina.

\section{Materials and Methods}

Double transgenic mice with inducible expression of BDNF in photoreceptors. Transgenic mice that carry the reverse tetracycline transactivator under control of the photoreceptor-specific promoters for rhodopsin (rho/rtTA mice, line $\mathrm{D}$ ) or interphotoreceptor retinoid-binding protein (IRBP/rtTA mice, line K) have been generated and characterized (Chang et al., 2000). We previously crossed these mice with transgenic mice that have the tetracycline response element (TRE) coupled to a full-length cDNA for vascular endothelial growth factor (VEGF) to generate double transgenic mice with doxycycline-inducible expression of VEGF in photoreceptors (Ohno-Matsui et al., 2002). To produce mice with inducible expression of BDNF, we coupled the TRE to a full-length cDNA for rat BDNF (generously provided by Dr. Freda Miller, McGill University, Montreal, Canada), which had been demonstrated previously to cause expression of bioactive BDNF in transgenic mice (Causing et al., 1997). TRE/BDNF transgenic mice were generated and screened for Tg incorporation by PCR of DNA extracted from 0.5 to $1 \mathrm{~cm}$ segments of tails digested overnight at $55^{\circ} \mathrm{C}$ in $500 \mu \mathrm{l}$ of $50 \mathrm{~mm}$ Tris, $\mathrm{pH}$ 7.5, $100 \mathrm{~mm}$ EDTA, $400 \mathrm{~mm} \mathrm{NaCl}$, and $0.5 \%$ SDS containing $0.25 \mu \mathrm{g}$ of proteinase $\mathrm{K}$. PCR was performed with $0.5 \mu \mathrm{g}$ of tail DNA and primers specific for the TRE/BDNF transgene ( $5^{\prime}$-CCATGGGTTACACGAAGGAAG-3' and 5' GACCTTGCACAGATAGCG-3 $3^{\prime}$ ) at an annealing temperature of $58^{\circ} \mathrm{C}$. Five founders were obtained and crossed with $\mathrm{C} 57 \mathrm{BL} / 6$ mice to generate five independent lines of TRE/BDNF transgenic mice. Double transgenic IRBP/rtTA-TRE/VEGF mice achieve higher levels of VEGF expression in the retina than rho/rtTA-TRE/VEGF mice (Ohno-Matsui et al., 2002), and therefore for this study we crossed IRBP/rtTA mice with mice from each of the TRE/BDNF lines to generate five IRBP/rtTA-TRE/BDNF lines. Double transgenic mice were identified by PCR of tail DNA. The TRE/BDNF transgene was identified using the primers listed above, and the IRBP/rtTA transgene was identified as described previously (Chang et al., 2000), using rtTA primer (5'-GTTTACCGATGCCCTTGGAATTGACGAGT-3') and IC40 primer ( $5^{\prime}$-GATGTGGCGAGATGCCCTTGGAATTGACGAGT-3'). Mice that were hemizygous at each transgene locus were used in all experiments.

Assessment of inducible expression of BDNF in IRBP/rtTA-TRE/BDNF mice. Adult double hemizygous mice from each of the five IRBP/rtTA$\mathrm{TRE} / \mathrm{BDNF}$ lines were treated with $2 \mathrm{mg} / \mathrm{ml}$ doxycycline in their drinking water or maintained on normal drinking water; after 2 weeks, mice were killed, retinas were dissected, and retinal RNA was isolated using the guanidine isothiocyanate method (Chomczynski and Sacchi, 1987). Reverse transcription (RT) was performed with $1 \mu \mathrm{g}$ of total RNA using the an RT-PCR kit from Invitrogen (Gaithersburg, MD), and PCR was performed on aliquots of the $\mathrm{CDNAs}$ at an annealing temperature of $58^{\circ} \mathrm{C}$ using primers (5'-CCATGGGTTACACGAAGGAAG-3' and 5'-GACCTTGCACAGATAGCG-3') that specifically amplify a 481 bp product of rat BDNF sequence. Mouse S16 ribosomal protein primers (5'-CACTGCAAACGGGGAAATGG-3' and 5' -TGAGATGGACTGTCGGATGG-3') were used to provide an internal control for the amount of template in the PCRs. Titration was performed to ensure that PCRs were performed in the linear range of amplification.

Immunohistochemical staining for BDNF. Mice were killed, and eyes were removed and frozen in optimum cutting temperature embedding compound (OCT; Miles, Elkhart, IN). Ocular frozen sections $(10 \mu \mathrm{m})$ were dried with cold air for $5 \mathrm{~min}$, fixed in freshly prepared $4 \%$ paraformaldehyde in $0.05 \mathrm{M} \mathrm{PBS}$ at $4^{\circ} \mathrm{C}$ for $30 \mathrm{~min}$, and rinsed with Tris-buffered saline (TBS) for $10 \mathrm{~min}$. Endogenous peroxidases were inhibited by a 10 min incubation with $0.75 \% \mathrm{H}_{2} \mathrm{O}_{2}$ in PBS. After two 10 min incubations in $0.1 \%$ Triton $\mathrm{X}-100$ in $50 \mathrm{~mm}$ TBS, nonspecific binding sites were blocked by incubating sections with $3 \%$ normal goat serum (NGS) in $0.1 \%$ Triton X-100 for $1 \mathrm{hr}$ at room temperature. Sections were incubated with 1:200 rabbit polyclonal anti-human BDNF antibody (Santa Cruz Biotechnology, Santa Cruz, CA) in 1\% NGS/PBS for $48 \mathrm{hr}$ at $4^{\circ} \mathrm{C}$. Control sections were processed without primary antibody or with primary antibody that had been preincubated with a human BDNF blocking peptide (sc-546P; Santa Cruz Biotechnology). After two rinses with TBS, sections were incubated for $1 \mathrm{hr}$ at room temperature with secondary antibody, 1:200 goat anti-rabbit IgG coupled to horseradish peroxidase (Vector Laboratories, Burlingame, CA). Slides were incubated with stable diaminobenzidine tetrahydrochloride (Invitrogen) containing $0.03 \%$ nickel ammonium chloride. Sections were rinsed in TBS and deionized water; dehydrated by passing them through a step gradient consisting of 70, 95, and $100 \%$ ethanol and xylene; and then mounted with Cytoseal XYL mounting medium (Richard-Alan Scientific, Kalamazoo, MI). The stained sections were examined under a Nikon (Tokyo, Japan) microscope and captured as digital files with a Nikon Digital Still Camera DXM1200.

Triple hemizygous IRBP/rtTA-TRE/BDNF-Q344ter mice. Transgenic mice that express truncated rhodopsin attributable to a mutation resulting in a stop codon at position 344, referred to as Q344ter mice, develop degeneration of rod photoreceptors starting at approximately postnatal day 10 (P10) and completed by approximately P21 (Sung et al., 1994). Mice homozygous for the Q344ter transgene were crossed with double homozygous IRBP/rtTA-TRE/BDNF mice to give litters of triple hemizygous IRBP/rtTA-TRE/BDNF-Q344ter mice that were randomly divided into doxycycline-treated $(\mathrm{DOX}+)$ and untreated $(\mathrm{DOX}-)$ litters. Mothers of DOX + litters were given drinking water containing $2 \mathrm{mg} / \mathrm{ml}$ doxycycline at $\mathrm{P} 0$, and the pups were given $0.1 \mathrm{ml}$ containing $15 \mu \mathrm{g} / \mathrm{gm}$ body weight of doxycycline every other day by gavage starting at P5. Pups from untreated litters were given $0.1 \mathrm{ml}$ of vehicle by gavage every other day starting at P5. Some mice were killed at P16, and the thickness of the outer nuclear layer (ONL) was measured as described below. At P21, mice were weaned, and some double transgenic mice were maintained on drinking water containing $2 \mathrm{mg} / \mathrm{kg}$ doxycycline for long-term studies, whereas others were maintained on normal drinking water to provided matched controls. The remainder of the double transgenic mice and all of the triple transgenic mice had ERGs performed at P21, and then the mice were killed. Some eyes were used to assess BDNF mRNA in the retina by RT-PCR as described above, some eyes were used for BDNF immunohistochemistry as described above, and some eyes were used to measure the thickness of the ONL. For the long-term studies reported in this manuscript, ERGs were performed on double transgenic mice that received 3 months of doxycycline and on matched controls.

Prolonged hyperoxia in double hemizygous IRBP/rtTA-TRE/BDNF mice. Exposure of adult mice to $70 \%$ oxygen for $>1$ week results in photoreceptor degeneration (Yamada et al., 1999, 2001). At P21, double hemizygous IRBP/rtTA-TRE/BDNF mice from the same litters were divided into untreated groups and groups treated with $2 \mathrm{mg} / \mathrm{ml}$ doxycycline in their drinking water. Both groups were exposed to $70 \%$ oxygen for 2 weeks and then killed. Some eyes were used to measure BDNF mRNA in the retina by RT-PCR, and the remainder were used for BDNF immunohistochemistry or measurement of the thickness of the ONL.

Measurement of ONL thickness. The 12:00 meridian of eyes was marked with a suture, and the eyes were then fixed in $0.1 \mathrm{M}$ phosphate buffer $(\mathrm{PB})$, $\mathrm{pH} 7.6$, containing $4 \%$ paraformaldehyde and $20 \%$ sucrose at $4^{\circ} \mathrm{C}$ for 2 $\mathrm{hr}$, rinsed in $0.1 \mathrm{M} \mathrm{PB}$ containing $20 \%$ sucrose overnight at $4^{\circ} \mathrm{C}$, and frozen in 1:2 OCT:PB containing 20\% sucrose. Ten micrometers of serial frozen sections perpendicular to the 12:00 meridian were cut from the superior border of the cornea and iris to the inferior border, a distance of $\sim 750 \mu \mathrm{m}$ (Fig. 1A). Every fifth section (total, 15) was used for measurements (unless it was poor quality, in which case an adjacent good quality section was used). Sections on either side, but not including the optic nerve, were used. The sections were stained with hematoxylin and examined with an Axioskop microscope (Zeiss, Thornwood, NY), and images 

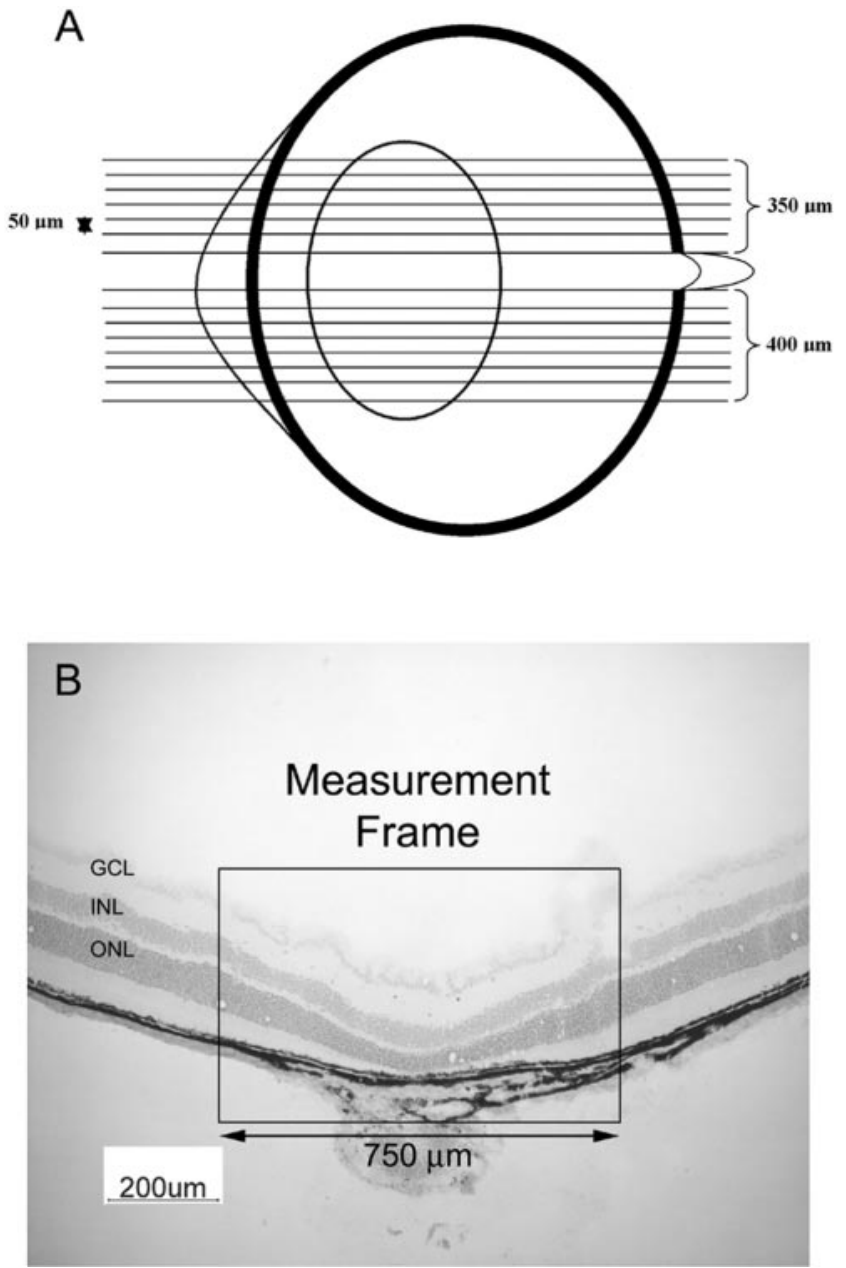

Figure 1. Measurement of ONL thickness in the posterior pole of eyes by image analysis. $A$, Schematic drawing illustrating ocular sections used for image analysis. The superior pole of eyes was marked at the time of enucleation, and eyes were embedded with the superior pole at the top. Ten micrometer sections were cut from the superior border to the inferior border of the cornea, a distance of $\sim 750 \mu \mathrm{m}$. Sections were stained with hematoxylin, and image analysis was performed on every fifth section (unless it was poor quality, in which case an adjacent good quality section was used), resulting in 15 measurements per eye that were averaged to give a single experimental value. $B$, Stained sections were viewed with an Axioskop microscope, and the image was digitized with a three $C C D$ camera and frame grabber. The posterior $750 \mu \mathrm{m}$ of the ONL was outlined on each section, and the area was calculated by image analysis. The INL and the $\mathrm{GCL}$ are seen above the $\mathrm{ONL}$.

were digitalized using a three-charge-coupled device (CCD) color video camera (IK-TU40A; Toshiba, Tokyo, Japan) and a frame grabber. With the investigator masked as to group, Image-Pro Plus software (Media Cybernetics, Silver Spring, MD) was used to outline $750 \mu \mathrm{m}$ of the outer nuclear layer spanning the posterior pole of the retina on that section (Fig. 1B). The mean of the measurements from the 15 sections from each eye was used as a single experimental value. Images for display were captured with a Nikon microscope equipped with Nikon Digital Still Camera DXM1200.

Recording and analysis of ERGs. The investigator that performed ERGs was masked with respect to treatment group of the mice. ERGs were recorded on seven DOX + and four DOX - triple hemizygous IRBP/ rtTA-TRE/BDNF-Q344ter mice. To assess for a potential effect of increased BDNF expression on retinal function, recordings were made on nine DOX + and 10 DOX - double hemizygous IRBP/rtTA-TRE/BDNF mice. Starting on the day of birth, mothers of DOX + mice were treated with $2 \mathrm{mg} / \mathrm{ml}$ doxycycline in their drinking water, and pups were given 15 $\mu \mathrm{g} / \mathrm{gm}$ body weight of doxycycline by gavage every other day between P5 and P21. At P21, mice were dark-adapted for a standardized $12 \mathrm{hr}$ period

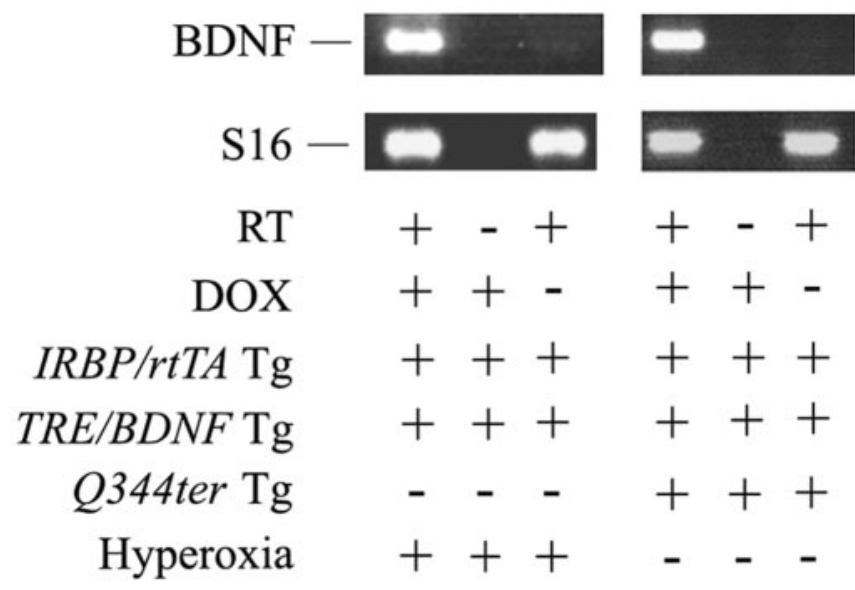

Figure 2. Doxycycline-induced expression of rat BDNF mRNA in double transgenic mice. Adult mice that carried the IRBP/rtTA Tg and the TRE/BDNF Tg were treated with DOX + or DOX - and exposed to 70\% oxygen. After $14 \mathrm{~d}$, the mice were killed and retinal RNA was isolated. Some litters of mice that carried the above two Tgs plus the Q344ter mutant rhodopsin Tg were untreated (DOX -), whereas for DOX + litters, mothers were treated with drinking water containing $2 \mathrm{mg} / \mathrm{ml}$ doxycycline at $\mathrm{P} 0$, and the pups were given $0.1 \mathrm{ml}$ containing 15 $\mu \mathrm{g} / \mathrm{gm}$ body weight of doxycycline every other day by gavage starting at P5. At P21, the mice were killed and retinal RNA was isolated. PCR was performed on $1 \mu \mathrm{g}$ of retinal RNA either with or without previous treatment with RT. In the presence of RT, there was an amplicon for S16 ribosomal protein $\mathrm{mRNA}$ in samples from DOX + and DOX - mice, but an amplicon for rat BDNF mRNA occurred only in samples from DOX + mice.

overnight, and ERG recordings were performed using the Espion ERG Diagnosys (Diagnosys LLL, Littleton, MA). All manipulations were done with dim red light illumination. Beginning at the same time each morning, mice were anesthetized by intraperitoneal injection of $25 \mu \mathrm{l} / \mathrm{gm}$ body weight of Avertin (Aldrich, Milwaukee, WI) and diluted 1:39 in PBS. Each cornea was anesthetized with a drop of $0.5 \%$ proparacaine hydrochloride (Alcon Labs, Forth Worth, TX), and pupils were dilated with 1\% mydriacyl ophthalmic solution (Alcon Labs). Mice were placed on a pad heated to $37^{\circ} \mathrm{C}$, and platinum electrodes were placed on each cornea after application of gonioscopic prism solution (Alcon Labs). The reference electrode was placed subcutaneously in the anterior scalp between the eyes, and the ground electrode was inserted into the tail. Electrode impedance was balanced for each eye pair measured. The head of the mouse was placed in a standardized position in a ganzfeld bowl illuminator that ensured equal illumination of the eyes. Simultaneous recordings from both eyes were made for 11 intensity levels of white light ranging from -3 to $1.40 \log \mathrm{cd}-\mathrm{s} / \mathrm{m}^{2}$. The Espion ERG machine measures the ERG response six times at each flash intensity and records the average value. The maximum a-wave and b-wave ERG response amplitude recorded for an eye at a particular stimulus intensity was used as the experimental value for the eye.

Statistical comparisons. ERG a-wave and b-wave amplitudes were compared by ANOVA with the data for right and left eyes averaged, and a log transformation for normality was applied before the analysis. Two separate models were fit to the a-wave and the b-wave data. The first model included data from all 3 weeks, and the second model included only the data for 3 weeks of the double Tg and 3 months of DOX-treated mice. For the 3 weeks of a-wave and b-wave data, there was a significant three-way interaction between flash intensity, genotype, and treatment (i.e., the treatment effect varied significantly by flash intensity and genotype; $p=$ 0.017 for a-wave; $p<0.001$ for b-wave). Hence, linear contrasts were used to compare DOX versus no DOX at each combination of flash intensity and genotype.

For the 3 months of double transgenic data, there were significant effects for flash intensity and treatment time (i.e., a-wave and b-wave amplitudes differed by both flash intensity and by time), but there were no significant treatment effects after controlling for flash intensity and treatment time. There were no significant interactions between any of the three experimental variables, intensity, time, or treatment. In particular, the tests for differing treatment effect by intensity or time were not sig- 

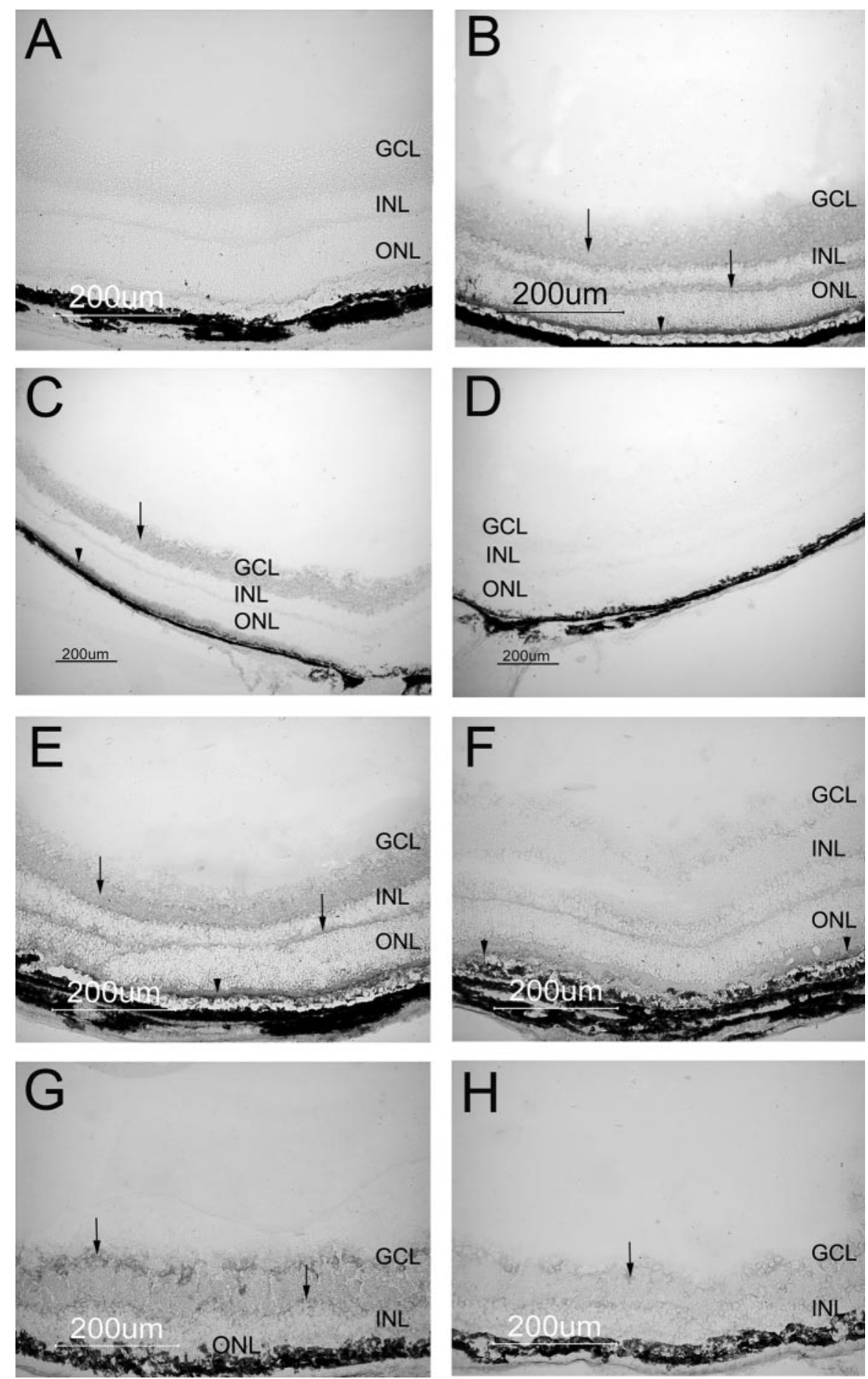

Figure 3. Immunohistochemistry for BDNF in doxycycline-treated or untreated double transgenic mice. Adult mice that carried the IRBP/rtTA Tg and the TRE/BDNFTg were treated with DOX+ or DOX - and exposed to $70 \%$ oxygen. After $14 \mathrm{~d}$, the mice were killed and the eyes were frozen in OCT embedding compound. Some litters of mice that carried the above two Tgs plus the Q344ter Tg were untreated, whereas for DOX + litters, mothers were treated with drinking water containing $2 \mathrm{mg} / \mathrm{ml} \mathrm{doxycycline} \mathrm{at} \mathrm{P0}$, and the pups were given $0.1 \mathrm{ml}$ containing $15 \mu \mathrm{g} / \mathrm{gm}$ body weight of doxycycline every other day by gavage starting at P5. At P21, the mice were killed and the eyes were frozen in OCT. Ten micrometer frozen sections were immunohistochemically stained for BDNF. $A$, A frozen section from a wild-type C57BL/6 mouse shows no staining for BDNF with the parameters for staining that were used. B, DOX+ double transgenic IRBP/rtTA-TRE/BDNF mice showed staining for BDNF in the inner retina, primarily in the inner and outer plexiform layers (arrows) and intense staining of photoreceptor inner segments (arrowhead). C, Sections from another DOX+ double transgenic showed a pattern of staining for BDNF that was similar to that seen in B, with strong staining in the inner plexiform layer (arrow) and photoreceptor inner segments (arrowhead). On a serial section, the staining was eliminated by preincubation of primary antibody with a BDNF peptide (D). E, Double transgenic /RBP/rtTA-TRE/BDNF mice put in $70 \%$ oxygen and treated with doxycycline for $14 \mathrm{~d}$ showed staining for BDNF in the inner and outer plexiform layers (arrows) and intense staining of photoreceptor inner segments (arrowhead). $F$, A region of peripheral retina from a DOX - double transgenic IRBP/rtTA-TRE/BDNFmouse that was put in 70\% oxygen for $14 \mathrm{~d}$ showed no staining for BDNF in the inner retina. There appears to be some faint staining in photoreceptors in some areas (arrowhead), suggesting that there may be low-level expression of BDNF in the absence of doxycycline. G, At P21, D0X + IRBP/rtTA-TRE/BDNF-rho/Q344ter triple transgenic mice showed staining for BDNF in ganglion cells (arrow) and the inner plexiform layer (bottom arrow) and mild staining elsewhere in the inner retina. H, At P21, DOX - IRBP/rtTA-TRE/BDNF-rho/Q344ter triple transgenic mice showed possible faint staining for BDNF in the inner retina, suggesting possible low-level expression of BDNF in the absence of doxycycline. 
nificant. Therefore, linear contrasts were not used to compare DOX versus no DOX at each combination of flash intensity and genotype.

For the oxidative damage model, the comparison of the ONL area between DOX-treated and untreated mice was done by a $t$ test. For the inherited retinal degeneration model, two time points were investigated, and the area of the ONL in the posterior pole for each time point and treatment was compared by ANOVA. Areas were averaged across the locations before the analysis. Linear contrasts were used to compare treatments within each level of time and vice versa.

\section{Results \\ Doxycycline-inducible expression of BDNF in double hemizygous IRBP/rtTA-TRE/BDNF mice}

Five independent TRE/BDNF transgenic lines were obtained and used to generate five IRBP/rtTA-TRE/BDNF double transgenic lines. Adult double hemizygous mice from each of the five lines were given normal drinking water or water supplemented with $2 \mathrm{mg} / \mathrm{ml}$ doxycycline. After 2 weeks, retinal levels of rat BDNF mRNA were measured by RT-PCR. Two lines showed expression of rat BDNF mRNA in the absence of doxycycline. One of the other three lines that showed minimal detectable expression in the absence of doxycycline showed higher doxycycline-induced expression of BDNF mRNA than the other two lines and was selected for subsequent experiments. Mice from that line exposed to hyperoxia and treated with doxycycline for 2 weeks showed high expression of BDNF mRNA, and those exposed to hyperoxia and not treated with doxycycline had a barely detectable band for rat BDNF mRNA, which is not easily seen in Figure 2 (left column). Triple transgenic IRBP/rtTA-TRE/BDNFQ344ter mice also showed barely detectable rat BDNF mRNA in the retina when not treated with doxycycline and high levels of BDNF mRNA when treated with doxycycline (Fig. 2, right column).

The parameters used for immunohistochemical staining for BDNF showed no staining in the retinas of wild-type C57BL/6 mice (Fig. 3A). After $14 \mathrm{~d}$ of administration of $2 \mathrm{mg} / \mathrm{ml}$ doxycycline in their drinking water, double transgenic IRBP/rtTA-TRE/BDNF mice showed staining for BDNF in the inner retina, primarily in the inner and outer plexiform layers, and intense staining of photoreceptor inner segments (Fig. $3 B, C$ ). With the IRBP promoter driving expression of rtTA, BDNF is produced in photoreceptors but is then secreted and spreads throughout the retina. The staining was completely eliminated by preincubation with a BDNF peptide (Fig. $3 D$ ), demonstrating the specificity of the staining for BDNF. The staining was not eliminated by prolonged exposure to hyperoxia (Fig. 3E) or by expression of the Q344ter transgene (Fig. 3G), suggesting that increased levels of BDNF are maintained in those settings, making it possible to DOX + mice $(E)$.
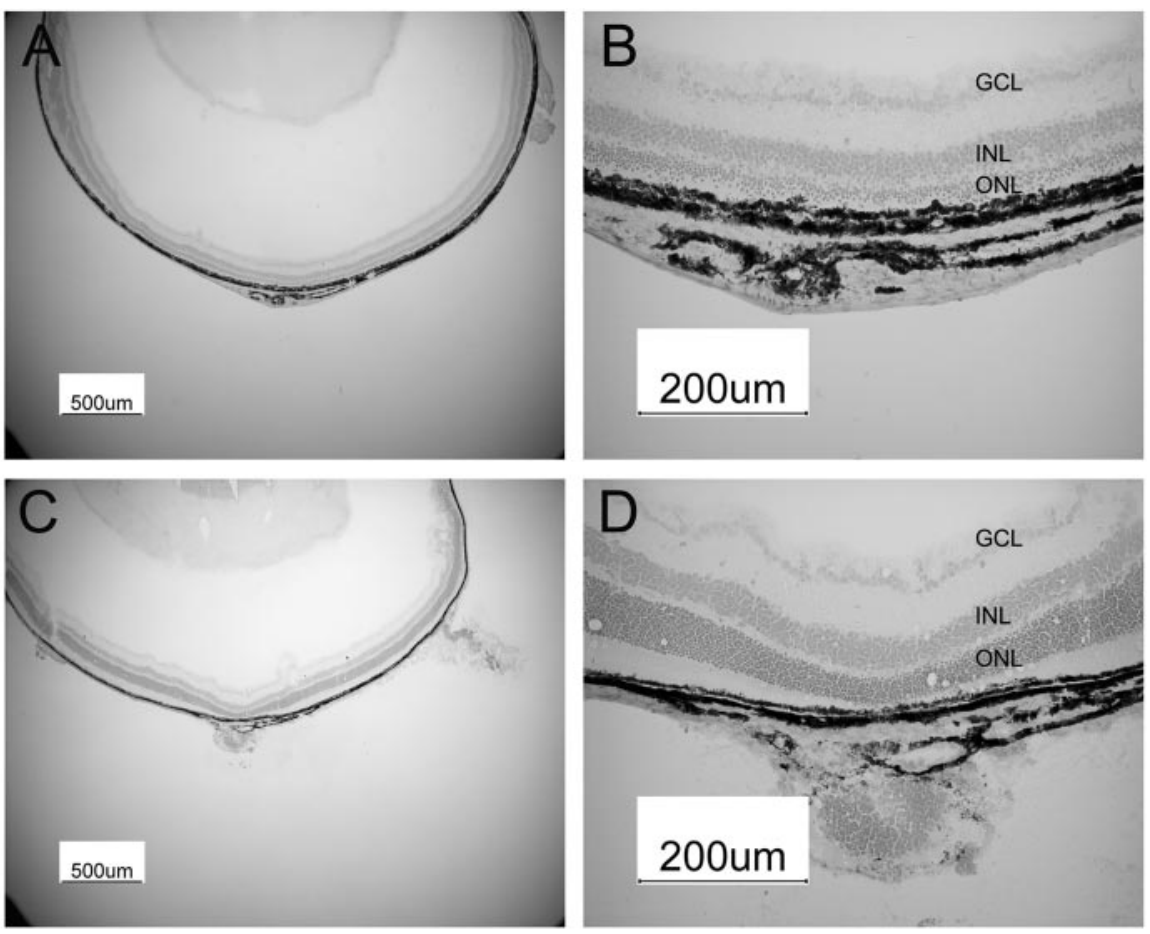

E

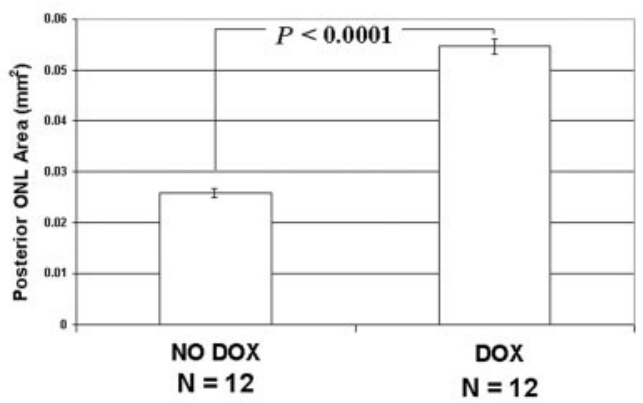

Figure 4. Expression of BDNF decreases hyperoxia-induced photoreceptor cell death. Adult mice that carried the IRBP/rtTA Tg and the TRE/BDNFTg were treated with DOX + or DOX - and exposed to 70\% oxygen. After $14 \mathrm{~d}$, the mice were killed and the eyes were frozen in OCT embedding compound. Ten micrometer frozen sections spanning the posterior $750 \mu \mathrm{m}$ of the retina were stained with hematoxylin. Sections from DOX - mice $(A, B)$ showed marked thinning of the ONL, which is made up of photoreceptor nuclei throughout the posterior region of the retina. In the low-magnification view shown in $A$, note that the 0 NL is thinner

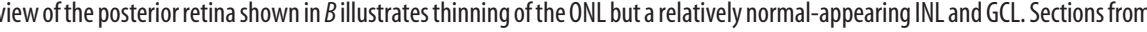
+ mice $(C, D)$ showed a normal-appearing $0 \mathrm{NL}$ both posteriorly and anteriorly, very similar to the $0 \mathrm{NL}$ seen in mice not and Methods and illustrated in Figure 1, showed significantly smaller ONL area in the posterior poles of DOX - mice compared with

assess the effects of BDNF on photoreceptor survival in those models.

BDNF decreases hyperoxia-induced photoreceptor cell death Exposure of adult mice to $70 \%$ oxygen for 2 or 3 weeks results in thinning of the ONL in the posterior pole (Yamada et al., 1999). This is consistent with a previous report of the damaging effects of hyperoxia on photoreceptors, presumably because of oxidative damage (Noell, 1955). Double hemizygous IRBP/rtTA-TRE/ BDNF mice that did not receive doxycycline (DOX-) during a 2 week period that they were exposed to hyperoxia showed substantial reduction in thickness of the outer nuclear layer in the posterior pole of the retina (Fig. $4 A, B$ ). In contrast, double hemizygous IRBP/rtTA-TRE/BDNF mice that had their drinking wa- 

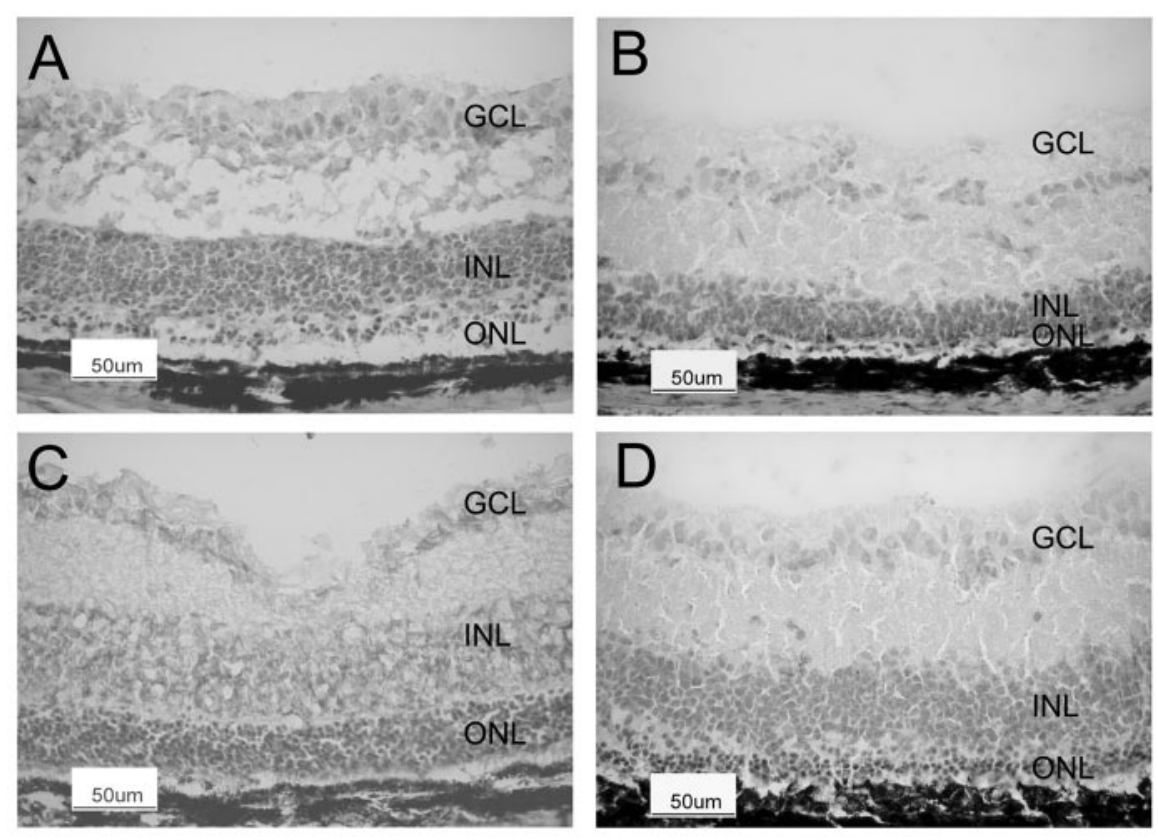

\section{E P16}
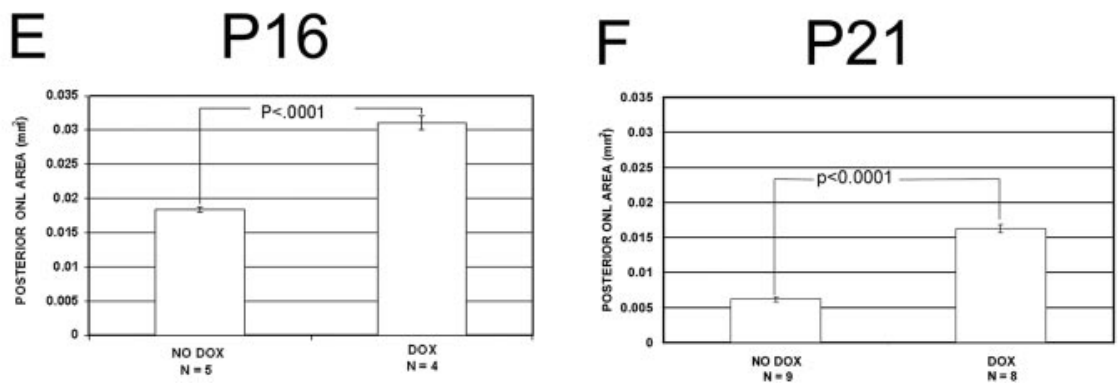

Figure 5. Expression of BDNF delays mutant rhodopsin-induced photoreceptor cell death. Mothers of DOX + triple transgenic IRBP/rtTA-TRE/BDNF-rho/Q344ter mice were treated with water supplemented with $2 \mathrm{mg} / \mathrm{kg}$ doxycycline, and starting at P5, the pups were treated every other day with $0.1 \mathrm{ml}$ containing $15 \mu \mathrm{g} / \mathrm{gm}$ body weight of doxycycline. Control DOX - triple transgenic mice and their mothers did not receive any doxycycline. At P16, DOX - mice $(A)$ showed severe photoreceptor degeneration with only a few rows of nuclei remaining in the $0 N L$. In contrast, $D O X+$ triple transgenic mice $(B)$ showed a much thicker ONL. The INL and $\mathrm{GCL}$ were comparable in DOX + and DOX - mice. At P21, DOX - mice $(C)$ showed almost a complete loss of photoreceptors, with only one row of nuclei in the ONL. There was also thinning of the INL. At P21, DOX + mice (D) showed several rows of nuclei in the $0 \mathrm{NL}$ and a normal-appearing INL and GCL. Measurements of the area of the ONL in the posterior pole for each time point and treatment were compared by ANOVA. Areas were averaged across the locations before the analysis. There was a statistically significant interaction between treatment and time in this model ( $p=0.0038)$. Linear contrasts were used to compare treatments with each level of time and vice versa. The treatment comparisons at both P16 $(E)$ and P21 $(F)$ were highly statistically significant ( $p<0.0001$ for both times). In addition, the time comparisons with each treatment (i.e., P16 vs P21 for DOX and P16 vs P21 for no DOX) were also highly statistically significant ( $p<0.0001$ for both comparisons).

ter supplemented with $2 \mathrm{mg} / \mathrm{ml}$ doxycycline (DOX + ) during 2 weeks of exposure to hyperoxia had preservation of the outer nuclear layer to the point that it was normal in appearance (Fig. $4 C, D)$. The average area of the outer nuclear layer in the posterior pole of the retina $(750 \times 750 \mu \mathrm{m})$ was significantly greater in eyes of mice treated with DOX + compared with DOX - mice (Fig. $4 E)\left(0.0550 \pm 0.0017\right.$ vs $\left.0.0258 \pm 0.0010 \mathrm{~mm}^{2} ; p<0.0001\right)$.

\section{BDNF delays photoreceptor cell death from Q344ter mutant rhodopsin}

Delivery of doxycycline to neonatal mice is more difficult than its delivery to adult mice. In breast milk of mothers given $2 \mathrm{mg} / \mathrm{ml}$ doxycycline in their drinking water, nursing pups obtain insufficient doxycycline to cause detectable transgene expression. Subcutaneous injections of doxycycline provide adequate levels for transgene induction (Ohno-Matsui et al., 2002) but induce scar- ring, which is particularly problematic in neonates and precludes giving more than a few injections. Therefore, a strategy was devised whereby mothers of newborn triple transgenic IRBP/rtTA-TRE/BDNFrho/Q344ter mice were given water supplemented with $2 \mathrm{mg} / \mathrm{ml}$ doxycycline, and starting at P5, pups were given $0.1 \mathrm{ml}$ containing $15 \mu \mathrm{g} / \mathrm{gm}$ body weight of doxycycline by gavage every other day. For control DOX - litters, mothers were given normal water and the pups were given vehicle by gavage starting at P5. At P16, DOX - triple hemizygous mice showed severe photoreceptor degeneration, with only a few rows of nuclei remaining in the ONL (Fig. 5A). In contrast, DOX + triple hemizygous mice showed a substantially thicker ONL (Fig. 5B). Image analysis confirmed that the average area of the ONL in ocular sections of the posterior pole of the retina was significantly greater in DOX + mice compared with DOXmice (Fig. 5E) (0.0311 \pm 0.0007 vs $0.0184 \pm 0.0003 \mathrm{~mm}^{2} ; p<0.0001$ by linear contrasts). The inner nuclear layer (INL) and ganglion cell layer (GCL) were comparable in DOX + and DOX - mice. At P21, DOX - mice showed an almost complete loss of photoreceptors, with only one row of nuclei remaining in the ONL (Fig. 5C). There was also thinning of the inner nuclear layer. At P21, DOX+ mice showed several rows of nuclei in the ONL (Fig. 5D), and image analysis showed significantly greater ONL area compared with DOX - mice (Fig. 5F) $(0.0163 \pm 0.0002$ vs $0.0062 \pm 0.0001$ $\mathrm{mm}^{2} ; p<0.0001$ by linear contrasts). The DOX + mice also had normal-appearing inner nuclear and ganglion cell layers.

\section{BDNF preserves retinal function in mice expressing mutant rhodopsin} Recordings of ERGs were performed to determine whether overexpression of BDNF in the retina has a deleterious effect on retinal function and whether photoreceptors rescued from mutant rhodopsin-induced cell death were functioning. Litters of newborn double transgenic IRBP/rtTA-TRE/BDNF or triple transgenic IRBP/rtTA-TRE/BDNF-rho/Q344ter mice were either left untreated with doxycycline and given only vehicle by gavage or treated with doxycycline by the regimen described above. At P21, mice were dark adapted for $12 \mathrm{hr}$ and ERGs were recorded from each eye after each of 11 incremental flashes of white light ranging from -3 to $1.40 \log \mathrm{cd}-\mathrm{s} / \mathrm{m}^{2}$. DOX + double transgenic mice showed normal-appearing retinas and normal ERG wave forms, with a-waves and b-waves that increased in amplitude with increasing light intensity (Fig. 6A). Compared with DOX - mice (Fig. $6 B$ ), there was no significant difference in a-wave or b-wave amplitudes or implicit times at any stimulus intensity (Fig. 6E, G, Table 1), suggesting that increased expression of BDNF in the retina for 3 weeks does not disturb retinal 
function as assessed by ERGs. ERG amplitudes were also measured in mice with increased expression of BDNF in the retina for 3 months and showed no significant differences from matched control mice (Table 1). Pooling across flash intensities, both groups of older mice had significantly larger a-wave $(p=0.0014)$ and b-wave $(p=0.0041)$ amplitudes than those seen in 3-week-old mice.

DOX - triple transgenic IRBP/rtTATRE/BDNF-rho/Q344ter mice showed nonrecordable ERGs at all stimulus intensities and almost a complete loss of photoreceptors, with only one row of nuclei remaining in the ONL (Fig. 6D). In contrast, $\mathrm{DOX}+$ triple transgenic mice still had several rows of nuclei remaining in the ONL at P21 and had recordable ERGs (Fig. 6C). At moderate and high stimulus intensities, $\mathrm{DOX}+$ triple transgenic mice had significantly greater a-wave and b-wave amplitudes than DOX - triple transgenic mice (Fig. 6F, Table 1), indicating that expression of BDNF resulted in preservation of some retinal function. ERG amplitudes were reduced in DOX+ triple transgenic mice compared with double transgenic mice, as expected from the reduction of photoreceptors. However, implicit times were similar between the two groups (Fig. $6 H$ ), suggesting that the remaining photoreceptors in DOX+ triple transgenic mice were functioning fairly well.

\section{Discussion}

In this study, we have demonstrated that increased expression of BDNF in photoreceptors provides strong protection against oxidative injury-induced photoreceptor cell death and incomplete, but still substantial, protection from death caused by mutant rhodopsin. The delay in photoreceptor cell death from mutant rhodopsin is accompanied by maintenance of ERGs with decreased a-wave and b-wave amplitudes but normal implicit times, suggesting preservation of function of surviving photoreceptors. These data support a role for $\mathrm{BDNF}$ as a potential therapeutic agent for treatment of retinal degenerations.

Our results differ from those obtained by intravitreous injection of BDNF in Q344ter mice (LaVail et al., 1998) or cats with rod-cone dystrophy (Chong et al., 1999), which failed to show any evidence of a beneficial effect. It is likely that the brief increase in BDNF concentration in the retina resulting from an intravitreous injection is insufficient to promote survival of photoreceptors. In contrast to the lack of effectiveness of a brief pulse of BDNF, an intravitreous injection of CNTF delayed photoreceptor cell death in some mice with inherited retinal degenerations, and it was concluded that CNTF is a particularly promising candidate for treatment of retinal degenerations (LaVail et al., 1998; Chong et al., 1999). However, subsequent studies have suggested
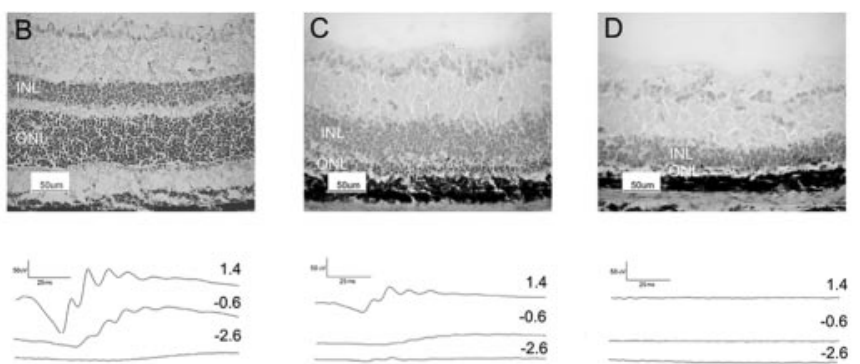

F

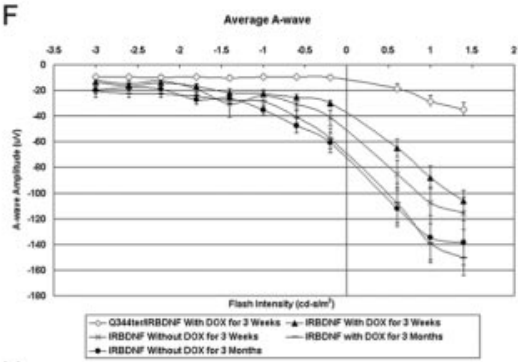

$\mathrm{H}$

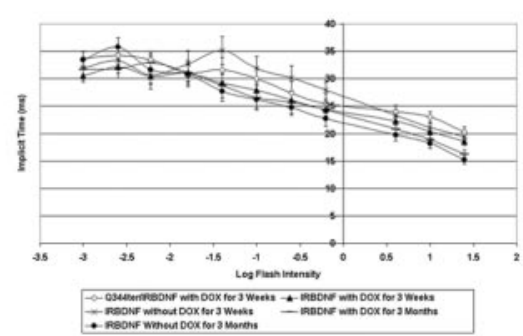

Figure 6. Expression of BDNF preserves retinal function in mice expressing mutant rhodopsin. Mothers of DOX + double transgenic IRBP/rtTA-TRE/BDNF or triple transgenic IRBP/rtTA-TRE/BDNF-rho/Q344ter mice were treated with water supplemented 列 Mothers of D0X - mice were given normal water, and the pups were not treated with doxycycline $(B, D)$. At P21, mice were dark 作 microscopy. A, DOX + double transgenic mice showed normal-appearing retinas and normal ERG wave forms with a-waves and $\mathrm{b}$-waves that increased in amplitude with increasing light intensity. $B, \mathrm{DOX}$ - transgenic mice showed normal-appearing retinas and ERG wave forms like those seen in DOX + double transgenic mice.C,DOX + triple transgenic IRBP/rtTA-TRE/BDNF-rho/Q344ter mice showed degeneration of photoreceptors but still had several rows of nuclei in the $0 N L$, whereas DOX - triple transgenic mice recordable ERGs at high light intensities $(C)$, indicating some preservation of function, whereas DOX - triple transgenic mice owed unrecordable ERGs at all light intensities $(D)$. The mean \pm SEM amplitude of the $b$-wave $(E)$ and the a-wave $(F)$ of the ERG group). DOX + and DOX - double transgenic mice showed nearly identical curves. DOX + triple transgenic IRBP/rtTA-TRE/BDNFrho/Q344ter mice showed increasing a-wave and b-wave amplitudes with increasing light intensities. The amplitudes were much less than those in double transgenic mice but much greater than those in DOX - triple transgenic mice.

that prolonged expression of CNTF in the retina may decrease ERG a-wave and b-wave amplitudes, suggesting a deleterious effect on retinal function (Liang et al., 2001). In this study, we found that expression of BDNF in the retina for 3 weeks or 3 months did not adversely affect retinal function as assessed by ERGs, and expression of BDNF in Q344ter mice resulted in both functional and morphologic rescue of photoreceptors. Therefore, contrary to previous impressions, BDNF may be a better candidate than CNTF as a therapeutic agent for some retinal degenerations.

We have shown previously that expression of FGF2 in photoreceptors provides strong protection from oxidative damageinduced death of photoreceptors (Yamada et al., 2001) very similar to that seen from expression of BDNF in this study. However, unlike BDNF, expression of FGF2 in photoreceptors provided no protection from mutant rhodopsin in Q344ter mice. The greater 
Table 1. Mean a-wave and b-wave ERG amplitudes after expression of BDNF in otherwise normal double transgenic mice and in triple transgenic mice that also express Q344ter mutant rhodopsin

\begin{tabular}{|c|c|c|c|c|c|c|}
\hline $\begin{array}{l}\text { Flash intensity (log } \\
\text { (d-s/m2) }\end{array}$ & $n$ & Genotype/treatment & a-wave $(\mu \mathrm{V})$ & $p$ value & b-wave $(\mu \mathrm{V})$ & $p$ value \\
\hline (1) -2.6 & 18 & Double Tg DOX+ for 3 weeks & $-14.8 \pm 1.64$ & & $45.8 \pm 4.23$ & \\
\hline (1b) -2.6 & 16 & Double Tg DOX + for 3 months & $-22.81 \pm 2.22$ & & $71.53 \pm 7.41$ & \\
\hline$(2)-2.6$ & 20 & Double Tg DOX - for 3 weeks & $-17.84 \pm 2.59$ & $p=0.59(1 \mathrm{vs} 2)$ & $47.9 \pm 8.37$ & $p=0.67(1$ vs 2$)$ \\
\hline$(2 b)-2.6$ & 12 & Double Tg DOX - for 3 months & $-17.72 \pm 1.66$ & $p=0.9^{*}(1 \mathrm{~b}$ vs $2 \mathrm{~b})$ & $81.76 \pm 8.49$ & $p=0.74^{*}(1 \mathrm{~b}$ vs $2 \mathrm{~b})$ \\
\hline (3) -2.6 & 14 & Triple Tg DOX + for 3 weeks & $-9.61 \pm 1.2$ & & $27.24 \pm 2.03$ & \\
\hline (4) -2.6 & 8 & Triple Tg DOX - for 3 weeks & $-14.42 \pm 4.49$ & $p=0.91(3$ vs 4$)$ & $25.08 \pm 2.74$ & $p=0.85(3$ vs 4$)$ \\
\hline (5) -0.6 & 18 & Double Tg DOX + for 3 weeks & $-28.25 \pm 3.4$ & & $171.04 \pm 13.52$ & \\
\hline$(5 b)-0.6$ & 16 & Double Tg DOX + for 3 months & $-40.71 \pm 4.44$ & & $220.34 \pm 20.03$ & \\
\hline (6) -0.6 & 20 & Double Tg DOX - for 3 weeks & $-27.13 \pm 1.93$ & $p=0.81(5$ vs 6$)$ & $152.98 \pm 14.95$ & $p=0.66(5$ vs 6$)$ \\
\hline (6b) -0.6 & 12 & Double Tg DOX - for 3 months & $-47.82 \pm 5.11$ & $p=0.9 *(5 b$ vs $6 b)$ & $238.29 \pm 19.31$ & $p=0.74^{*}(5 b$ vs $6 b)$ \\
\hline (7) -0.6 & 14 & Triple Tg DOX + for 3 weeks & $-9.45 \pm 0.89$ & & $53.41 \pm 3.79$ & \\
\hline (8) -0.6 & 8 & Triple Tg DOX - for 3 weeks & $-5.17 \pm 1.73$ & $p=0.024$ (7 vs 8$)$ & $11.61 \pm 2.64$ & $p=0.0002$ (7 vs 8$)$ \\
\hline (9) +1.4 & 18 & Double Tg DOX + for 3 weeks & $-113.38 \pm 8.98$ & & $254.44 \pm 16.16$ & \\
\hline (9b) +1.4 & 16 & Double Tg DOX + for 3 months & $-150.41 \pm 12.47$ & & $313.8 \pm 28.45$ & \\
\hline$(10)+1.4$ & 20 & Double Tg DOX - for 3 weeks & $-109.21 \pm 6.29$ & $p=0.99(9$ vs 10$)$ & $216.7 \pm 23.61$ & $p=0.58(9$ vs 10$)$ \\
\hline$(10 b)+1.4$ & 12 & Double Tg DOX - for 3 months & $-138.71 \pm 14.94$ & $p=0.9^{*}(9 \mathrm{~b}$ vs $10 \mathrm{~b})$ & $280.11 \pm 20.65$ & $p=0.74^{*}$ (9b vs $\left.10 \mathrm{~b}\right)$ \\
\hline$(11)+1.4$ & 14 & Triple Tg DOX+ & $-34.92 \pm 4.64$ & & $86.38 \pm 6.89$ & \\
\hline$(12)+1.4$ & 8 & Triple Tg DOX- & $-6.53 \pm 1.57$ & $p<0.0001$ (11 vs 12$)$ & $15.83 \pm 3.34$ & $p<0.0001$ (11 vs 12$)$ \\
\hline
\end{tabular}

Litters of IRBP/rtTA-TRE/BDNF mice (double Tg) were either treated with doxycycline (DOX+) to express BDNF or remained untreated with doxycycline, receiving only vehicle by gavage (DOX-) to serve as controls. For double Tg/DOX+ mice, mothers were given water supplemented with $2 \mathrm{mg} / \mathrm{kg}$ doxycycline, and starting at P5, the pups were given $0.1 \mathrm{ml}$ containing $15 \mu \mathrm{g}$ of doxycycline by gavage every other day. Litters of IRBP/rtTA-TRE/BDNF mice that also carried the Q344ter mutant rhodopsin transgene (triple Tg) were either treated with doxycycline (DOX+) as described above to express BDNF or remained untreated with doxycycline, receiving only vehicle by gavage (DOX -) to serve as controls. At P21, mice were anesthetized and ERGs were performed using 11 flash intensities (results for three displayed above: low,$-2.60 ;$ medium, $-0.60 ;$ high, 1.40). Compared with age-matched double Tg/DOX-mice, double Tg/DOX + mice that expressed ectopic BDNF in the retina for 3 weeks or 3 months showed no statistically significant differences (ANOVA) in mean a-wave or b-wave amplitudes at any of the flash intensities. Compared with triple Tg/DOX - mice, triple Tg/DOX+ mice that expressed ectopic BDNF in the retina had significantly greater mean a-wave and b-wave amplitudes at the two higher flash intensities.

* Treatment effect did not significantly differ by flash intensity or treatment time (3 weeks vs 3 months); hence, the $p$ value for treatment effect combining across flash intensities and treatment time is reported.

efficacy of BDNF and FGF2 in the model of retinal degeneration attributable to oxidative damage, compared with their effect in Q344ter mice with mutant rhodopsin-induced cell death, suggests that neurotrophic factors are likely to have different effectiveness in different types of retinal degeneration depending on the mechanisms of cell death. The greater benefit provided by BDNF compared with FGF2 in Q344ter mice could be attributable to the fact that BDNF activates different signaling pathways that more effectively block the initiation of a cell death program by mutant rhodopsin. Another possibility is that protection from oxidative damage requires initiation of signaling pathways only within photoreceptors, whereas survival factor-induced signaling in other cells in the retina that act indirectly on photoreceptors is needed to rescue photoreceptors from mutant rhodopsin-induced cell death. Staining for FGF2 in rho/FGF2 transgenic mice is limited to photoreceptors, which is expected because FGF2 is not a secreted protein, whereas BDNF is a secreted protein and, as noted in Figure 2, when BDNF expression is induced in photoreceptors of IRBP/rtTA-TRE/BDNF double transgenic mice, BDNF staining occurs throughout the retina. Therefore, BDNF may stimulate other retinal cells to produce survival signals that act on photoreceptors. This possibility is consistent with the observation that intravitreous injections of BDNF result in immunohistochemical staining for c-fos or pERK (phosphorylated extracellular signal-regulated kinase) in Muller cells and some other retinal cells but not photoreceptors (Wahlin et al., 2000, 2001). Furthermore, most studies that have attempted to localize tyrosine receptor kinase (trk) B receptors in the retina have demonstrated that they are present on ganglion cells and cells in the inner nuclear layer, including Muller cells, but not on photoreceptors (Perez and Caminos, 1995; Rickman and Brecha, 1995; Ugolini et al., 1995; Rohrer et al., 1999; Harada et al., 2000). However, one study with rodents has suggested that green-red-sensitive cones have trkB receptors (DiPolo et al., 2000), and in chicken photoreceptors, trkB and trkC expression is suppressed by darkness and upregulated by light exposure (Okazawa et al., 1994). Regardless of whether BDNF acts directly on photoreceptors or indirectly through other retinal cells and/or RPE cells, which also express trkB (Hackett et al., 1998), BDNF influences photoreceptor development and function (Rickman and Bowes Rickman, 1996; Rohrer et al., 1999) as well as survival. In an experimental model of retinal detachment, eyes injected with BDNF had longer and more organized rod outer segments (Lewis et al., 1999), providing another example of a BDNF-induced effect on photoreceptors.

Although additional studies are needed to investigate the mechanism by which BDNF influences the survival, development, and functioning of photoreceptors, this study demonstrates that BDNF has potential as a therapeutic agent for retinal degeneration. Additional studies using an inducible transgenic system are needed to compare the effects of BDNF with other survival factors, particularly glial cell line-derived neurotrophic factor (GDNF) and neurotrophic factor-3 (NT-3), because their receptors have been identified on photoreceptors (Harada et al., 2000, 2002). In fact, it has been demonstrated that GDNF has considerable potential as a therapeutic agent for retinal degeneration, because expression of GDNF in the retina by gene transfer results in delayed photoreceptor degeneration and maintenance of ERG a-wave and b-wave amplitudes in a transgenic rat model of primary rod cell degeneration (Sanftner et al., 2001). Although intravitreous injections of NT-3 showed little efficacy in mouse models of inherited degeneration (LaVail et al., 1998), as is true for BDNF, continuous expression of NT-3 may be needed to achieve morphologic and functional benefit. It would be useful to use the same inducible expression system to compare efficacy of each of these survival factors in the same models and to determine whether coexpression of multiple factors provides additive or synergistic benefit. This strategy provides a feasible first step for identification of an agent or mixture of agents for clinical trials.

\section{References}

Causing CG, Gloster A, Aloyz R, Bamji SX, Chang E, Fawcett J, Kuchel G, Miller FD (1997) Synaptic innervation density is regulated by neuronderived BDNF. Neuron 18:257-267. 
Cayouette M, Gravel C (1997) Adenovirus-mediated gene transfer of ciliary neurotrophic factor can prevent photoreceptor degeneration in the retinal degeneration (rd) mouse. Hum Gene Ther 8:423-430.

Cayouette M, Behn D, Sendtner M, Lachapelle P, Gravel C (1998) Intraocular gene transfer of ciliary neurotrophic factor prevents death and increases responsiveness of rod photoreceptors in the retinal degeneration slow mouse. J Neurosci 18:9282-9293.

Chang MA, Horner JW, Conklin BR, DePinho RA, Bok D, Zack DJ (2000) Tetracycline-inducible system for photoreceptor-specific gene expression. Invest Ophthalmol Vis Sci 41:4281-4287.

Chomczynski P, Sacchi N (1987) Single-step method of RNA isolation by acid guanidinium thiocyanate-phenol-chloroform extraction. Anal Biochem 162:156-159.

Chong NHV, Alexander RA, Waters L, Barnett KC, Bird AC, Luthert PJ (1999) Repeated injections of a ciliary neurotrophic factor analogue leading to long-term photoreceptor survival in hereditary retinal degeneration. Invest Ophthalmol Vis Sci 40:1298-1305.

Connell G, Bascom R, Molday L, Reid D, McInnes RR, Molday RS (1991) Photoreceptor peripherin is the normal product of the gene responsible for retinal degeneration in the rds mouse. Proc Natl Acad Sci USA 88:723-726.

DiPolo A, Cheng L, Bray GM, Aguayo AJ (2000) Colocalization of TrkB and brain-derived neurotrophic factor proteins in green-red-sensitive cone outer segments. Invest Ophthalmol Vis Sci 41:401-421.

Faktorovich EG, Steinberg RH, Yasumura D, Matthes MT, LaVail MM (1990) Photoreceptor degeneration in inherited retinal dystrophy delayed by fibroblast growth factor. Nature 347:83-86.

Faktorovich EG, Steinberg RH, Yasumura D, Matthes MT, La Vail MM (1992) Basic fibroblast growth factor and local injury protect photoreceptors from light damage in the rat. J Neurosci 12:3554-3567.

Hackett SF, Friedman Z, Freund J, Schoenfeld C, Curtis R, DiStefano PS, Campochiaro PA (1998) A splice variant of trkB and brain-derived neurotrophic factor oar co-expressed in retinal pigmented epithelial cells and promote differentiated characteristics. Brain Res 789:201-212.

Harada T, Harada C, Nakayama N, Okuyama S, Yoshida D, Kohsaka S, Matsuda H, Wada K (2000) Modification of glial-neuronal cell interactions prevents photoreceptor apoptosis during light-induced retinal degeneration. Neuron 26:533-541.

Harada T, Harada C, Kohsaka S, Wada E, Yoshida K, Ohno S, Mamada H, Tanaka K, Parada LF, Wada K (2002) Microglia-Muller glia cell interactions control neurotrophic factor production during light-induced retinal degeneration. J Neurosci 22:9228-9236.

LaVail MM, Unoki K, Yasumura D, Matthes MT, Yancopoulos G, Steinberg RH (1992) Multiple growth factors, cytokines, and neurotrophins rescue photoreceptors from the damaging effects of constant light. Proc Natl Acad Sci USA 89:11249-11253.

LaVail MW, Yasamura D, Matthes MT, Lau-Villacorta C, Unoki K, Sung CK, Steinberg RH (1998) Protection of mouse photoreceptors by survival factors in retinal degenerations. Invest Ophthalmol Vis Sci 39:592-602.

Lewis GF, Linberg KA, Geller SF, Guerin CJ, Fisher SK (1999) Effects of the neurotrophin brain-derived neurotrophic factor in an experimental model of retinal detachment. Invest Ophthalmol Vis Sci 40:1530-1544.
Liang F-Q, Aleman TS, Dejneka NS, Dudus L, Fisher KJ, Maguire AM, Jacobson SG, Bennett J (2001) Long-term protection of retinal structure but not function using RAAV: CNTF in animal models of retinitis pigmentosa. Mol Ther 4:461-472.

Noell WK (1955) Visual cell effects of high oxygen pressures. Fed Proc 14:107-108.

Ohno-Matsui K, Hirose A, Yamamoto S, Saikia J, Okamoto N, Gehlbach P, Duh EJ, Hackett SF, Chang M, Bok D, Zack DJ, Campochiaro PA (2002) Inducible expression of vascular endothelial growth factor in photoreceptors of adult mice causes severe proliferative retinopathy and retinal detachment. Am J Pathol 160:711-719.

Okazawa H, Kamei M, Imafuku I, Kanazawa I (1994) Gene regulation of trk $\mathrm{B}$ and trk $\mathrm{C}$ in the chick retina by light/darkness exposure. Oncogene 9:1813-1818.

Perez M-TR, Caminos E (1995) Expression of brain-derived neurotrophic factor and of its functional receptor in neonatal and adult rat retina. Neurosci Lett 183:96-99.

Rickman DW, Bowes Rickman C (1996) Suppression of trkB expression by antisense oligonucleotides alters a neuronal phenotype in the rod pathway of the developing rat retina. Proc Natl Acad Sci USA 93:12564-12569.

Rickman DW, Brecha NC (1995) Expression of the proto-oncogene, trk, receptors in the developing rat retina. Vis Neurosci 12:215-222.

Rohrer B, Korenbrot JI, LaVail MM, Reichardt LF, Xu B (1999) Role of neurotrophin receptor trkB in the maturation of rod photoreceptors and establishment of synaptic transmission to the inner retina. J Neurosci 19:8919-8930.

Sanftner LHM, Abel H, Hauswirth WW, Flannery JG (2001) Glial cell line derived neurotrophic factor delays photoreceptor degeneration in a transgenic rat model of retinitis pigmentosa. Mol Ther 4:622-629.

Sung C-H, Makino C, Baylor D, Nathans J (1994) A rhodopsin gene mutation responsible for autosomal dominant retinitis pigmentosa results in a protein that is defective in localization to the photoreceptor outer segment. J Neurosci 14:5818-5833.

Travis GH, Brennan MB, Danielson PE, Kozak CA, Sutcliffe JG (1989) Identification of a photoreceptor-specific mRNA encoded by the gene responsible for retinal degeneration slow $(r d s)$. Nature 338:70-73.

Ugolini G, Cremisi F, Maffei L (1995) TrkA, trkB, p75 mRNA expression is developmentally regulated in the rat retina. Brain Res 704:121-124.

Wahlin K, Campochiaro PA, Zack DJ, Adler R (2000) Neurotrophic factors cause activation of intracellular signaling pathways in Muller cells and other cells of the inner retina, but not photoreceptors. Invest Ophthalmol Vis Sci 41:927-936.

Wahlin KJ, Adler R, Zack DJ, Campochiaro PA (2001) Neurotrophic factor signaling in normal and degenerating rodent retinas. Exp Eye Res 73:693-701.

Yamada H, Yamada E, Hackett SF, Ozaki H, Okamoto N, Campochiaro PA (1999) Hyperoxia causes decreased expression of vascular endothelial growth factor and endothelial cell apoptosis in adult retina. J Cell Physiol 179:149-156.

Yamada H, Yamada E, Ando A, Esumi N, Bora N, Saikia J, Sung C-H, Zack DJ, Campochiaro PA (2001) FGF2 decreases hyperoxia-induced cell death in mice. Am J Pathol 159:1113-1120. 